\title{
Habenula deep brain stimulation for intractable schizophrenia: a pilot study
}

\author{
Yuhan Wang, MS, ${ }^{1}$ Chencheng Zhang, MD, PhD, ${ }^{1}$ Yingying Zhang, MSc, ${ }^{1}$ Hengfen Gong, MD, ${ }^{2}$ \\ Jun Li, PhD, ${ }^{1}$ Haiyan Jin, MD, ${ }^{3}$ Dianyou Li, MD, PhD, ${ }^{1}$ Dengtang Liu, MD, PhD, ${ }^{4}$ and \\ Bomin Sun, MD, PhD'
}

\begin{abstract}
${ }^{1}$ Department of Functional Neurosurgery, Ruijin Hospital, Shanghai Jiao Tong University School of Medicine; '2Department of Psychiatry, Pudong Mental Health Center, Shanghai; ${ }^{3}$ Department of Psychiatry, Ruijin Hospital, Shanghai Jiao Tong University School of Medicine; and ${ }^{4}$ First-episode Schizophrenia and Early Psychosis Program, Division of Psychotic Disorders, Shanghai Mental Health Center, Shanghai Jiao Tong University School of Medicine, Shanghai, China
\end{abstract}

Schizophrenia is a psychiatric disorder associated with significant morbidity and mortality. Although antipsychotic medications and electroconvulsive therapy can be used to manage the clinical symptoms of schizophrenia, a substantial portion (10\%-30\%) of patients do not clinically respond to these treatments or cannot tolerate the side effects. Recently, deep brain stimulation (DBS) has emerged as a promising safe and effective therapeutic intervention for various psychiatric disorders. Here, the authors explore the utility of DBS of the habenula (HB) in the clinical management of 2 young adult male patients with severe, chronic, and treatment-resistant schizophrenia. After HB DBS surgery, both patients experienced improvements in clinical symptoms during the first 6 months of treatment. However, only 1 patient retained the clinical benefits and reached a favorable outcome at 12-month follow-up. The symptoms of the other patient subsequently worsened and became so profound that he needed to be hospitalized at 10-month follow-up and withdrawn from further study participation. It is tentatively concluded that HB DBS could ultimately be a relatively safe and effective surgical intervention for certain patients with treatment-resistant schizophrenia.

https://thejns.org/doi/abs/10.3171/2020.4.FOCUS20174

KEYWORDS habenula; deep brain stimulation; schizophrenia

$\mathrm{S}$ CHIZOPHRENIA is a severe mental disorder with a lifelong prevalence of approximately $1 \% .^{1}$ The disorder is associated with significant morbidity and mortality. Symptoms of schizophrenia can be divided into "positive" symptoms (e.g., delusions, hallucinations); "negative" symptoms (e.g., flattened affect, loss of motivation); and cognitive symptoms (e.g., attention deficits, impaired working memory). To date, antipsychotic medication is the most widely used primary treatment for controlling the symptoms of patients with schizophrenia. However, $10 \%-$ $30 \%$ of patients display a poor clinical response to antipsychotic medications. Also, some studies have reported that more than $30 \%$ of patients treated with antipsychotic medication still experience some psychotic episodes or chronic residual clinical symptoms over the treatment course, despite the marked initial clinical improvement with phar- macotherapy. ${ }^{2}$ Electroconvulsive therapy (ECT) has also been used as a therapeutic intervention for severe, acute, and medication-resistant cases of schizophrenia, ${ }^{3}$ but the mode of action of ECT remains diffuse and elusive, and this intervention may even impair a patient's frontotemporal functions. ${ }^{4}$ Accordingly, there is a need for alternative treatment options for patients who suffer from severe, chronic, and medication-refractory schizophrenia.

In recent years, deep brain stimulation (DBS) has been found to be useful in the clinical management of several treatment-resistant psychiatric diseases, particularly obsessive-compulsive disorder (OCD), ${ }^{5}$ Tourette's syndrome, ${ }^{6}$ addiction, ${ }^{7}$ and depression. ${ }^{8}$ However, to date, only 3 clinical trials of DBS for treatment-refractory schizophrenia have been registered (ClinicalTrials.gov [accessed February 2020]). One clinical trial (NCT01725334) targeted the

ABBREVIATIONS ACC = anterior cingulate cortex; DBS = deep brain stimulation; $E C T=$ electroconvulsive therapy; $\mathrm{HB}=$ habenula; NAcc = nucleus accumbens; $\mathrm{PANSS}=$ Positive and Negative Syndrome Scale; VTA = volume of tissue activated.

SUBMITTED February 29, 2020. ACCEPTED April 7, 2020.

INCLUDE WHEN CITING DOI: 10.3171/2020.4.FOCUS20174. 
nucleus accumbens (NAcc) to manage negative symptoms but was withdrawn due to difficulties with recruiting eligible patients. Another trial (NCT02361554) is focused on the substantia nigra pars reticulata to treat positive and cognitive symptoms, but this trial is ongoing and currently in the initial phase of patient recruitment. The only clinical trial ${ }^{9}$ that has been completed (NCT02377505) examined the therapeutic effects of DBS of the NAcc and subgenual anterior cingulate cortex (ACC). The primary clinical endpoint used was a $25 \%$ improvement in the Positive and Negative Syndrome Scale (PANSS) total score after DBS treatment. The basic result was that 2 of the 3 patients who had received NAcc stimulation and 2 of the 4 patients treated with DBS of the subgenual ACC reached the primary endpoint. ${ }^{9}$ However, some contradictory results showed that high-voltage NAcc DBS could induce psychotic symptoms in vulnerable individuals. ${ }^{10}$

Accordingly, there have been few studies conducted on the clinical utility of DBS in managing medicationrefractory schizophrenia. Also, no consensus seems to exist about the specific brain structures, networks, and functions that could be the clinically optimal targets in DBS treatment for schizophrenia. Experimental data from animal studies are equivocal in this regard. For example, in rodent models of schizophrenia, a variety of brain structures, including the medial prefrontal cortex, NAcc, mediodorsal thalamus, and ventral hippocampus, were all found to be effective as targets of stimulation. ${ }^{11-13}$

In this study, we explored whether DBS of the habenula (HB) would be safe and similarly effective in treating medication-resistant schizophrenia. The HB is an epithalamic structure that modulates dopaminergic midbrain functions and serotonergic raphe nucleus activity. The HB is thought to play a key role in not only sleep and wakefulness but also the processing of aversive stimulus events, including pain, stress, and fear-provoking stimuli. Given its role in emotional processing, motivation, and dopaminergic function, it may be hypothesized that HB dysfunction contributes to the pathophysiology of schizophrenia. Indeed, in rodent models of schizophrenia, the metabolism of glucose in the lateral HB shows a significant increase after the administration of antipsychotic medications that have had their therapeutic effects in the animals. Such results indicate that the beneficial effects of antipsychotic medications are partially mediated by the restoration of lateral HB hypofunction in schizophrenia. ${ }^{14,15}$

Furthermore, a neuroimaging study reported the presence of substantial HB calcification in a group of 20 patients with schizophrenia. ${ }^{16}$ In addition, a postmortem study analyzed the brains of 13 patients with schizophrenia and 9 healthy control participants. ${ }^{17}$ The results showed the presence of markedly decreased capillary densities and reduced expression of the adenosine $5^{\prime}$-triphosphate (ATP)binding cassette transporter protein $\mathrm{ABCB} 1$ in the $\mathrm{HB}$ of patients. Other neuroimaging studies have also presented data indicating that abnormal HB structure and function contribute to the pathophysiology and clinical symptoms of schizophrenia. ${ }^{18,19}$ In this context, we initiated a pilot study to explore the safety and clinical effectiveness of bilateral DBS of the HB (HB DBS) in 2 patients with severe, chronic, and treatment-refractory schizophrenia.

\section{Methods}

\section{Study Participants}

We examined 2 male patients who met DSM-5 (Diagnostic and Statistical Manual of Mental Disorders) diagnostic criteria for schizophrenia and received bilateral $\mathrm{HB}$ DBS treatment. Table 1 presents several clinical characteristics of each patient. The study protocol was approved by the Ruijin Hospital Ethics Committee of the Shanghai Jiao Tong University School of Medicine. Written informed consent was obtained from each patient and their legal representatives before study participation.

\section{Case 1}

At the time of DBS surgery (2018), the patient was 26 years old. At the age of 17 years (2009), he started to experience auditory hallucinations, delusions of persecution, and babbling, as well as showing impulsive and abnormal behaviors. He was then diagnosed with schizophrenia at a local hospital. Between 2009 and 2018, he had been hospitalized 10 times and had accepted multiple treatment sessions with ECT. These interventions produced only mild and transient symptom relief. Moreover, he attempted but failed to clinically respond to multiple medications, including risperidone, clozapine, and quetiapine. Considering that his clinical symptoms were chronic, severe, and resistant to treatment, the patient became eligible for DBS treatment for schizophrenia.

Subsequently, he was referred to our hospital (June 2018) while taking olanzapine (10 mg/day) and lithium carbonate $(0.25 \mathrm{~g} /$ day $)$ in an attempt to control his symptoms, which now consisted of not only positive symptoms, such as auditory hallucinations and delusions, but also negative symptoms, such as blunted affect, poor attention, and lack of insight. After the patient was carefully evaluated by the hospital's DBS medical team, along with communication between the doctor and the patient and his parents, the decision was made to initiate bilateral HB DBS treatment.

\section{Case 2}

At the time of surgery, the patient was 21 years of age. In the past 4 years, he had experienced auditory hallucinations and delusions of persecution, and he exhibited destructive and aggressive behaviors. He also showed suicidal ideation. He was initially treated with quetiapine, lorazepam, and ECT, which temporarily alleviated his symptoms but were ultimately not successful due to poor patient compliance. As the patient grew older, his clinical symptoms gradually became more severe and disabling. Ultimately, he was admitted to our hospital (December 2018) while being on quetiapine ( $0.2 \mathrm{~g} /$ day), benzensol ( 4 $\mathrm{mg}$ /day), and ziprasidone (60 mg/day). Given the patient's clinically progressive deterioration, he and his parents accepted the therapeutic option to undergo HB DBS treatment in an effort to control his severe, chronic, and treatment-resistant schizophrenia symptoms.

\section{Clinical Outcome Assessment}

Clinical assessments were conducted before HB DBS surgery (baseline) and repeatedly during the study follow- 
TABLE 1. Clinical characteristics of each patient

\begin{tabular}{ccccccc}
\hline Case No. & Sex & $\begin{array}{c}\text { Duration of } \\
\text { Illness (yrs) }\end{array}$ & $\begin{array}{c}\text { Age at Onset of } \\
\text { Disease }(\mathrm{yrs})\end{array}$ & $\begin{array}{c}\text { Age at Surgery } \\
\text { (yrs) }\end{array}$ & $\begin{array}{c}\text { No. of } \\
\text { Hospitalizations }\end{array}$ & ECT \\
\hline 1 & Male & 9 & 17 & 26 & 10 & Yes \\
\hline 2 & Male & 4 & 17 & 21 & 4 & Yes \\
\hline
\end{tabular}

up period of 12 months. The frequency and timing of the outcome assessments slightly differed between the 2 patients (see Results). The primary clinical outcome was the severity of the patients' positive and negative symptoms, as assessed by using the PANSS total and scale scores. We also documented the frequency and nature of adverse side effects or complications associated with the HB DBS treatment.

\section{Surgical Procedure and Image Processing}

The implantation of the DBS leads (case 1: Model 3389, Medtronic; case 2: Model L302, PINS) was performed while the patients were under general anesthesia. A stereotactic frame was used to place the electrodes on the bilateral HB. According to the susceptibility-weighted imaging and quantitative susceptibility mapping in 3.0-T MRI, the $\mathrm{HB}$ was located in the region of the triangular depression of the third ventricle..$^{20,21}$ The stereotactic coordinates of the habenula region were $2 \mathrm{~mm}$ in front of the posterior commissure, $3-5 \mathrm{~mm}$ on lateral, and $0-1 \mathrm{~mm}$ above the anterior commissure-posterior commissure.

We used Lead-DBS version 2.0 software $^{22}$ to locate the electrode and compute the volumes of tissue activated (VTAs) of the stimulation parameters associated with the lowest PANSS total score. The location of the electrode and the VTA are presented in Figs. 1 and 2. According to the figures, the computed VTA contains the reconstructed HB area in both cases 1 and 2. In addition, the active contacts are all located in the HB area.

\section{Acute Electrical Stimulation}

One month after the surgery, we tested the acute HB DBS effects in both patients. Using a pulse width of 60 usec and frequencies of $60 \mathrm{~Hz}$ and $135 \mathrm{~Hz}$, we increased the voltage from $0 \mathrm{~V}$ to $10 \mathrm{~V}$ at the unit of $1 \mathrm{~V}$ in each contact. The increase in voltage was terminated when patients experienced intolerable side effects. Patients were tested while sitting in a chair and their heart rates were moni-
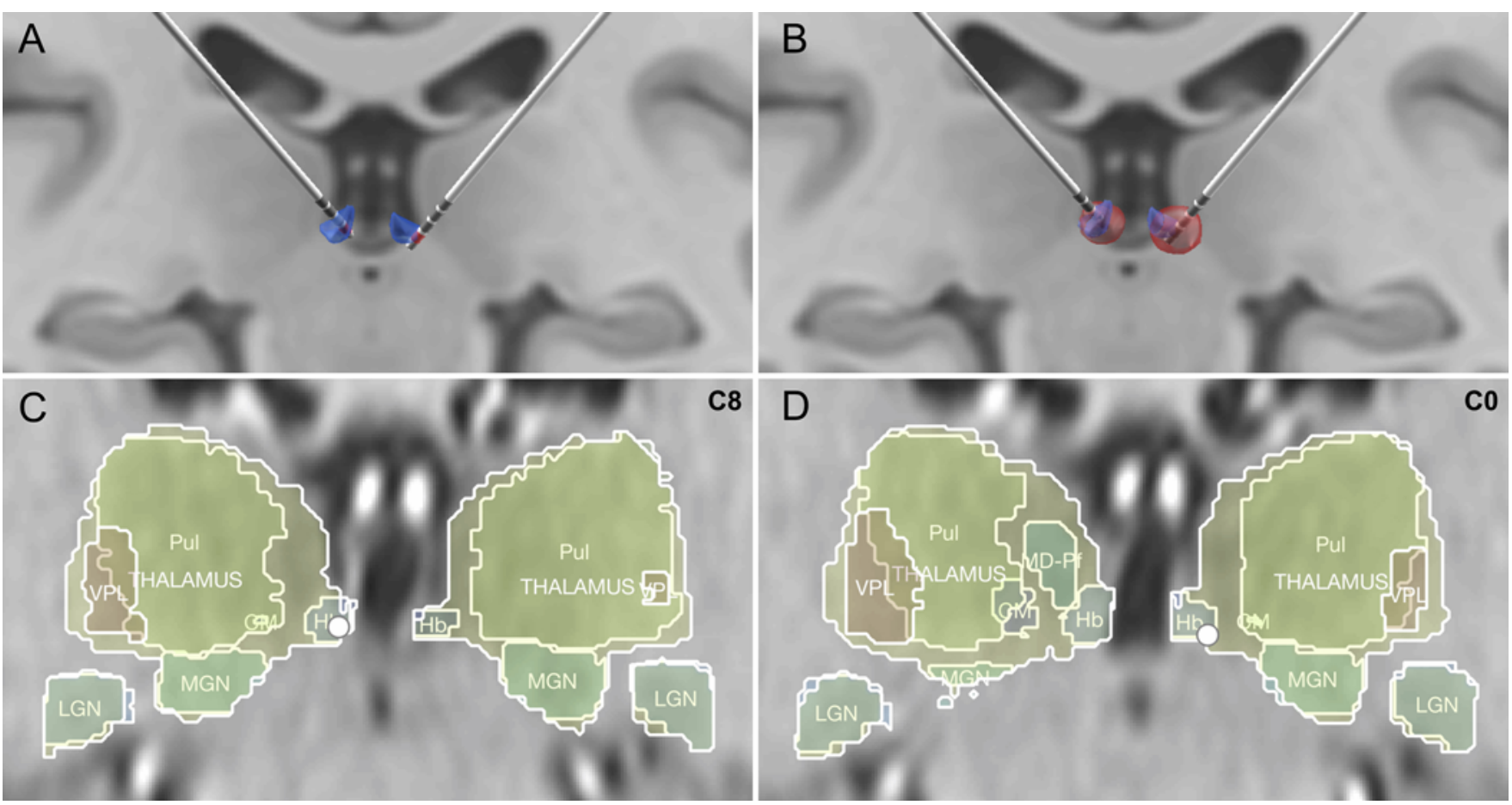

FIG. 1. Case 1. The location of electrodes and VTA. A: The blue area represents the reconstruction of bilateral $\mathrm{HB}(\mathrm{Hb})$, and the red dots represent the active contacts (left electrode: $\mathrm{C}+8-$; right electrode: $\mathrm{C}+0-$ ). B: The red area represents the VTA computed with the best stimulation parameters (left electrode: $2.0 \mathrm{~V}, 60 \mu \mathrm{sec}, 60 \mathrm{~Hz}, \mathrm{C}+8-$; right electrode: $2.5 \mathrm{~V}, 60 \mu \mathrm{sec}, 60 \mathrm{~Hz}, \mathrm{C}+0-$ ). $\mathrm{C}$ and $\mathrm{D}$ : The white dots represent the location of the active contacts (left electrode: $\mathrm{C}+8-;$ right electrode: $\mathrm{C}+0-$ ); the contact number is noted in the top right corner of each image. $\mathrm{CM}=$ centromedian nucleus; $\mathrm{LGN}=$ lateral geniculate nucleus; $\mathrm{MD}-\mathrm{Pf}=$ mediodorsal nucleus-parafascicular nucleus; $M G N=$ medial geniculate nucleus; $P$ ul = pulvinar nucleus; $V P L=$ ventral posterior lateral nucleus. 

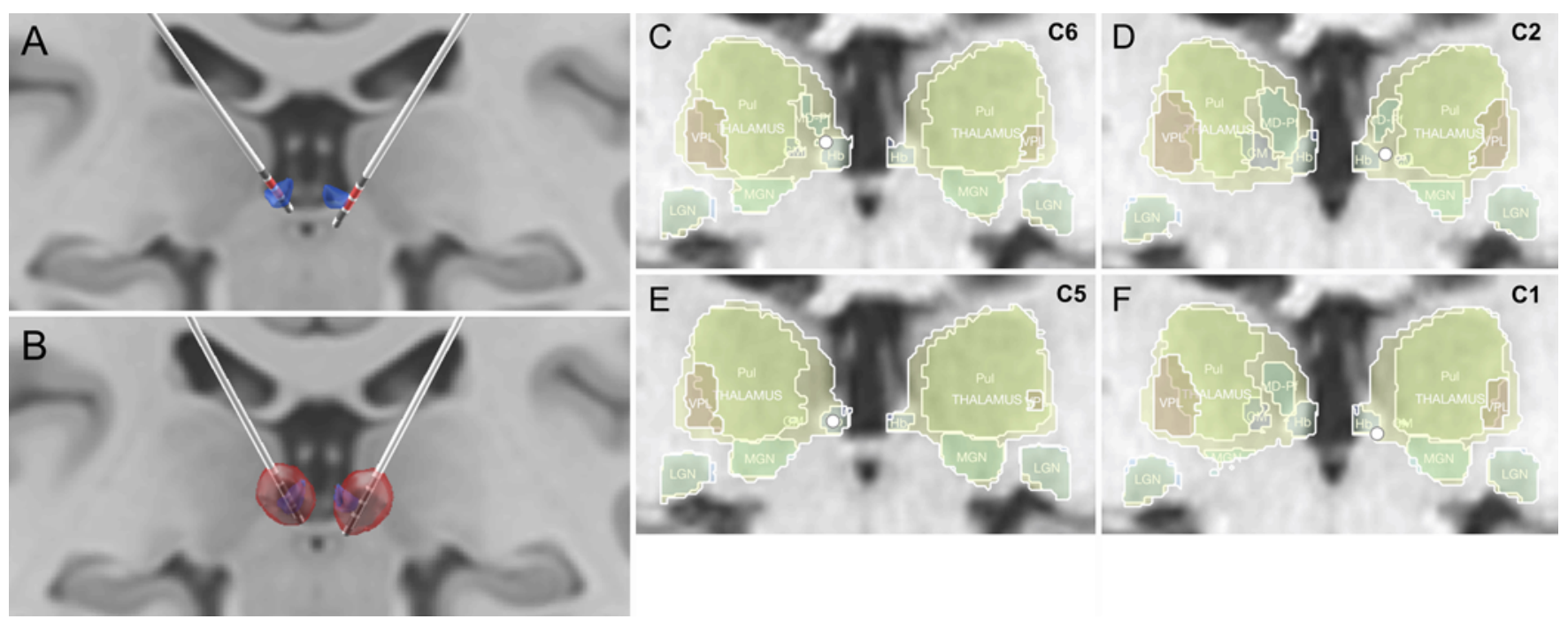

FIG. 2. Case 2. The location of electrodes and VTA. A: The blue area represents the reconstruction of bilateral HB, and the red dots represent the active contacts (left electrode: $\mathrm{C}+5-6-$; right electrode: $\mathrm{C}+1-2-)$. B: The red area represents the VTA computed with the best stimulation parameters (left electrode: $3.15 \mathrm{~V}, 80 \mu \mathrm{sec}, 135 \mathrm{~Hz}, \mathrm{C}+5-6$-; right electrode: $3.2 \mathrm{~V}, 60 \mu \mathrm{sec}, 135$ $\mathrm{Hz}, \mathrm{C}+1-2-)$. C-F: The white dot represents the location of the active contacts (left electrode: $\mathrm{C}+5-6-$; right electrode: $\mathrm{C}+1-2-$ ); the contact number is noted in the top right corner of each image. $\mathrm{CM}=$ centromedian nucleus; $\mathrm{LGN}=$ lateral geniculate nucleus; $\mathrm{MD}-\mathrm{Pf}=$ mediodorsal nucleus - parafascicular nucleus; $\mathrm{MGN}=$ medial geniculate nucleus; $\mathrm{Pul}=$ pulvinar nucleus; $\mathrm{VPL}=$ ventral posterior lateral nucleus.

tored. The programmer sat behind, one observer sat diagonally opposite to the patient, and another observer together with a camera sat $3 \mathrm{~m}$ in front of the patient (Video 1).

VIDEO 1. Acute electrical stimulation effect of HB DBS. Copyright Chencheng Zhang. Published with permission. Click here to view.

In this setting, the programmer could adjust the parameters in a manner unknown to the patient. Occasionally, the programmer provided some incorrect parameter information to the patient to check and identify the genuine effects of stimulation.

\section{Results}

Before HB DBS, both patients showed chronic and disabling schizophrenia symptoms, but the symptoms of the patient in case 1 (PANSS baseline scores: Positive, 13; Negative, 23; General, 38) were overall less severe than those seen in the patient in case 2 (PANSS baseline scores: Positive, 26; Negative, 41; General, 53).

\section{Case 1}

After HB DBS surgery, the patient in case 1 displayed improvements in his clinical symptoms over the first 7 months of stimulation, ranging from an $8.7 \%$ improvement in negative symptoms to a $31.6 \%$ improvement in general psychopathology (Table 2). The exception was that the severity of his positive symptoms first increased by $7.7 \%$ at 6-month follow-up and then decreased by $7.7 \%$ at 7-month follow-up. After 7 months of treatment, however, the patient's clinical state deteriorated, especially the severity of his positive symptoms. As a result, his intake of olanzapine was increased to $15 \mathrm{mg} /$ day (Table 2). Notwithstanding, his auditory hallucinations, delusions, incoherent thinking, poor self-insight, and disruptive and aggressive behaviors gradually recurred. At 10-month follow-up, he was hospitalized in a local hospital for aggravated psychotic symptoms. At that time, his positive symptoms were increased by approximately $69 \%$ when compared with his score at baseline. The decision was made to withdraw the patient

TABLE 2. Clinical outcomes during the 10 -month follow-up period in the patient in case 1

\begin{tabular}{ccccc}
\hline & \multicolumn{4}{c}{ PANSS Scores (\% improvement) } \\
\cline { 2 - 5 } Date of Assessment & Positive Scale & Negative Scale & General Psychopathology Scale & Total \\
\hline Baseline & 13 & 23 & 38 & 74 \\
\hline 6-mo FU & $14(-7.7 \%)$ & $19(17.4 \%)$ & $33(13.2 \%)$ & $66(10.8 \%)$ \\
\hline 7-mo FU & $12(7.7 \%)$ & $21(8.7 \%)$ & $26(31.6 \%)$ & $59(20.3 \%)$ \\
\hline 10-mo FU & $22(-69.2 \%)$ & $24(-4.3 \%)$ & $35(7.9 \%)$ & $81(-9.5 \%)$ \\
\hline
\end{tabular}

$\mathrm{FU}=$ follow-up.

Value in parentheses indicates the percent increase (positive values) or decrease (negative values) in the patient's

PANSS score from baseline to follow-up. 
TABLE 3. Clinical outcomes during the 12-month follow-up period in the patient in case 2

\begin{tabular}{ccccc}
\hline & \multicolumn{4}{c}{ PANSS Scores (\% improvement) } \\
\cline { 2 - 5 } Date of Assessment & Positive Scale & Negative Scale & General Psychopathology Scale & Total \\
\hline Baseline & 26 & 41 & 53 & 120 \\
\hline 1-mo FU & $23(11.5 \%)$ & $28(31.7 \%)$ & $30(43.4 \%)$ & $81(32.5 \%)$ \\
\hline 4-mo FU & $22(15.4 \%)$ & $34(17.1 \%)$ & $64(-20.8 \%)$ & $120(0)$ \\
\hline 6-mo FU & $21(19.2 \%)$ & $40(2.4 \%)$ & $43(18.9 \%)$ & $104(13.3 \%)$ \\
\hline 7-mo FU & $22(15.4 \%)$ & $33(19.5 \%)$ & $41(22.6 \%)$ & $96(20.0 \%)$ \\
\hline 12-mo FU & $12(53.8 \%)$ & $28(31.7 \%)$ & $42(20.8 \%)$ & $82(31.7 \%)$ \\
\hline
\end{tabular}

Value in parentheses indicates the percent increase (positive values) or decrease (negative value) in the patient's PANSS score from baseline to follow-up.

from the HB DBS treatment study. The DBS parameters used for this patient were $2.0 \mathrm{~V}, 60 \mu \mathrm{sec}$, and $60 \mathrm{~Hz}$ on the left side and $2.5 \mathrm{~V}, 60 \mu \mathrm{sec}$, and $60 \mathrm{~Hz}$ on the right side, which remained fixed throughout the follow-up.

\section{Case 2}

After HB DBS surgery, the patient's overall level of symptoms was markedly improved at 1-month follow-up, ranging from an $11.5 \%$ improvement in positive symptoms to a $43.4 \%$ improvement in general psychopathology (Table 3). The stimulation parameters used were $2.5 \mathrm{~V}, 60 \mu \mathrm{sec}$, and $60 \mathrm{~Hz}$ on the left side and $2.0 \mathrm{~V}, 60 \mu \mathrm{sec}$, and $60 \mathrm{~Hz}$ on the right side. At 4-month follow-up, he presented with a marked worsening of general psychopathology, possibly due to a prior DBS voltage change (from $2.5 \mathrm{~V}$ to $3.5 \mathrm{~V}$ on the left side). Subsequently, we increased his dose of quetiapine to $0.4 \mathrm{~g} /$ day for the remaining period of the study. From 4- to 12-month follow-up, the patient gradually regained most of the clinical benefits achieved at 1-month follow-up. It took us approximately 8 months to adjust and identify the DBS parameters $(3.15 \mathrm{~V}, 80 \mu \mathrm{sec}$, and $135 \mathrm{~Hz}$ on the left side and $3.2 \mathrm{~V}, 60 \mu \mathrm{sec}$, and $135 \mathrm{~Hz}$ on the right side) most suitable for this patient. At 12-month follow-up, the patient exhibited clinically significant improvements in the PANSS positive symptom score by $53.8 \%$, negative symptom score by $31.7 \%$, general psychopathology score by $20.8 \%$, and total score by $31.7 \%$ (Table 3 ). Additionally, the patient's parents had to stay with him all the time before the HB DBS surgery, but they could leave him alone at home without worries during the daytime at 12-month follow-up.

\section{Responses to Acute Electrical Stimulation}

In our 2 patients, 323 active trials were tested during which 255 induced an immediate response. The response rate was $81.7 \%$ (156/191) in case 1 and 75\% (99/132) in case 2 . The majority of responses consisted of numbness $(28.2 \%)$, change of heart rate $(26.7 \%)$, pain $(14.1 \%)$, dizziness $(5.5 \%)$, and eye closing (5.1\%). The fewest responses included discomfort (1.2\%), feelings of heaviness $(0.4 \%)$, and feelings of relaxation (0.4\%). The patients often reported feelings of relief or euphoria after their emergence from numbness or pain.

\section{Discussion}

In this pilot study, we evaluated the clinical utility of HB DBS in managing the clinical symptoms in 2 patients with severe, chronic, and treatment-refractory schizophrenia. The HB DBS treatment turned out to be relatively safe and was well tolerated in both patients. The results obtained concerning the clinical effectiveness were mixed. Although both patients experienced improvements in their clinical symptoms in the first 6 months of HB DBS treatment, only 1 patient (case 2 ) reached a favorable clinical outcome at 12-month follow-up. By contrast, the symptoms of the other patient (case 1) subsequently worsened and became so severe and disruptive that he had to be hospitalized at 10-month follow-up and could no longer participate in the study.

These results make it difficult to reach a general and robust conclusion about the clinical effectiveness of $\mathrm{HB}$ DBS for treatment-resistant schizophrenia. It is noteworthy, however, that the patient who experienced a favorable outcome presented with less severe clinical symptoms at baseline as compared with the symptom severity of the patient who experienced a poor outcome. Also, although the initial clinical symptoms became evident in both patients at the age of 17 years, the duration of illness at baseline was substantially shorter (4 vs 9 years) for the patient who achieved a favorable outcome at 12-month follow-up. These clinical observations raise the possibility that HB DBS could provide an effective treatment option for only those patients with refractory schizophrenia who are not so severely ill or who have not been ill for quite a long time at the start of the surgical treatment.

One previous study indicated that DBS of the NAcc or subgenual ACC could also be effective for some patients with treatment-resistant schizophrenia. ${ }^{9}$ In contrast to the adverse effect profiles observed in this prior study, however, no significant adverse side effects emerged in our 2 patients. Indeed, the acute stimulation tests revealed that the power of the stimulation needed to modulate the HB was relatively low. Thus, HB DBS could be a relatively safe and well-tolerated alternative surgical intervention for certain patients with treatment-resistant schizophrenia.

In addition, the present study provides some putative evidence for the hypothesis that HB hypofunction contributes to the pathophysiology of at least some cases of schizophrenia. After DBS of the HB, 1 patient showed a 
marked improvement in the overall severity of his symptoms at 12-month follow-up. Interestingly, his positive symptoms were particularly improved (by $53.8 \%$ ). It is commonly believed that the positive symptoms of schizophrenia are related to dopaminergic hyperfunction. In rodent models of schizophrenia, ${ }^{23-25}$ dopaminergic hyperfunction can be produced by HB damage via the fimbriaNAcc pathway. Similarly, dopaminergic activity can be reduced by administration of antipsychotic drugs (dopamine D2 receptor antagonists). ${ }^{26}$

Finally, it should be acknowledged that our pilot study of 2 cases is limited by various methodological problems, making it not possible to draw any firm conclusions about the potential clinical effectiveness of the HB as a novel DBS target for medically intractable schizophrenia. Yet, to date, the effects of DBS have been reported in only 9 patients, ${ }^{9,27}$ so our report provides additional clinical information relevant to DBS treatment for schizophrenia. Similarly, we present a reasonably clear picture of the procedure used, the sequence in which events occurred, and of the setting in which they took place. Furthermore, based on the findings from this pilot study, we believe that we can safely proceed with this DBS target for treatmentresistant schizophrenia. Notwithstanding, we did not control for possible sham effects of DBS, which are usually nonnegligible in psychiatric disorders, and hence could provide an alternative explanation of the clinical benefits seen in the patients. For further study, we intend to implement a double-blind DBS discontinue stage to differentiate therapeutic stimulation effects from sham effects.

\section{Conclusions}

We tentatively conclude that HB DBS may be a promising surgical therapeutic intervention for some cases of chronic, severe, and treatment-refractory schizophrenia. HB DBS may act locally to restore HB hypofunction and more globally to suppress dopaminergic hyperfunction in schizophrenia, which may be particularly effective for those patients with severe positive symptoms. The initiation of larger, well-controlled studies seems warranted to assess the utility of HB DBS as an alternative treatment option for schizophrenia.

\section{References}

1. Saha S, Chant D, Welham J, McGrath J. A systematic review of the prevalence of schizophrenia. PLoS Med. 2005;2(5):e141.

2. Kane JM, Agid O, Baldwin ML, et al. Clinical guidance on the identification and management of treatment-resistant schizophrenia. J Clin Psychiatry. 2019;80(2):18com12123.

3. Sanghani SN, Petrides G, Kellner CH. Electroconvulsive therapy (ECT) in schizophrenia: a review of recent literature. Curr Opin Psychiatry. 2018;31(3):213-222.

4. Fosse R, Read J. Electroconvulsive treatment: hypotheses about mechanisms of action. Front Psychiatry. 2013;4:94.

5. Denys D, Graat I, Mocking R, et al. Efficacy of deep brain stimulation of the ventral anterior limb of the internal capsule for refractory obsessive-compulsive disorder: a clinical cohort of 70 patients. Am J Psychiatry. 2020:177(3):265-271.

6. Xu W, Zhang C, Deeb W, et al. Deep brain stimulation for Tourette's syndrome. Transl Neurodegener. 2020;9:4.
7. Ma S, Zhang C, Yuan T, et al. Neurosurgical treatment for addiction: lessons from an untold story in China and a path forward. Natl Sci Rev. 2019;7(3):702-712.

8. Crowell AL, Riva-Posse P, Holtzheimer PE, et al. Longterm outcomes of subcallosal cingulate deep brain stimulation for treatment-resistant depression. Am J Psychiatry. 2019;176(11):949-956.

9. Corripio I, Roldán A, Sarró S, et al. Deep brain stimulation in treatment resistant schizophrenia: a pilot randomized crossover clinical trial. EBioMedicine. 2020;51:102568.

10. Graat I, Bergfeld IO, de Koning P, et al. Delusions following deep brain stimulation of the nucleus accumbens. Brain Stimul. 2019;12(3):770-771.

11. Perez SM, Shah A, Asher A, Lodge DJ. Hippocampal deep brain stimulation reverses physiological and behavioural deficits in a rodent model of schizophrenia. Int J Neuropsychopharmacol. 2013;16(6):1331-1339.

12. Mikell CB, McKhann GM, Segal S, et al. The hippocampus and nucleus accumbens as potential therapeutic targets for neurosurgical intervention in schizophrenia. Stereotact Funct Neurosurg. 2009;87(4):256-265.

13. Klein J, Hadar R, Götz T, et al. Mapping brain regions in which deep brain stimulation affects schizophrenia-like behavior in two rat models of schizophrenia. Brain Stimul. 2013;6(4):490-499.

14. Fakhoury M. The habenula in psychiatric disorders: more than three decades of translational investigation. Neurosci Biobehav Rev. 2017;83:721-735.

15. Dedeurwaerdere S, Wintmolders C, Vanhoof G, Langlois X. Patterns of brain glucose metabolism induced by phosphodiesterase 10A inhibitors in the mouse: a potential translational biomarker. J Pharmacol Exp Ther. 2011;339(1):210-217.

16. Sandyk R. Pineal and habenula calcification in schizophrenia. Int J Neurosci. 1992;67(1-4):19-30.

17. Bernstein H-G, Hildebrandt J, Dobrowolny H, et al. Morphometric analysis of the cerebral expression of ATP-binding cassette transporter protein ABCB1 in chronic schizophrenia: circumscribed deficits in the habenula. Schizophr Res. 2016;177(1-3):52-58.

18. Shepard PD, Holcomb HH, Gold JM. Schizophrenia in translation: the presence of absence: habenular regulation of dopamine neurons and the encoding of negative outcomes. Schizophr Bull. 2006;32(3):417-421.

19. Zhang L, Wang H, Luan S, et al. Altered volume and functional connectivity of the habenula in schizophrenia. Front Hum Neurosci. 2017;11:636.

20. Zhang C, Kim S-G, Li D, et al. Habenula deep brain stimulation for refractory bipolar disorder. Brain Stimul. 2019;12(5):1298-1300.

21. He N, Sethi SK, Zhang C, et al. Visualizing the lateral habenula using susceptibility weighted imaging and quantitative susceptibility mapping. Magn Reson Imaging. 2020;65:5561.

22. Horn A, Kühn AA. Lead-DBS: a toolbox for deep brain stimulation electrode localizations and visualizations. Neuroimage. 2015;107:127-135.

23. Lecourtier L, Kelly PH. Bilateral lesions of the habenula induce attentional disturbances in rats. Neuropsychopharmacology. 2005;30(3):484-496.

24. Lecourtier L, Deschaux O, Arnaud C, et al. Habenula lesions alter synaptic plasticity within the fimbria-accumbens pathway in the rat. Neuroscience. 2006;141(2):1025-1032.

25. Lecourtier L, Neijt HC, Kelly PH. Habenula lesions cause impaired cognitive performance in rats: implications for schizophrenia. Eur J Neurosci. 2004;19(9):2551-2560.

26. Amato D, Vernon AC, Papaleo F. Dopamine, the antipsychotic molecule: a perspective on mechanisms underlying antipsychotic response variability. Neurosci Biobehav Rev. 2018;85:146-159. 
27. Plewnia C, Schober F, Rilk A, et al. Sustained improvement of obsessive-compulsive disorder by deep brain stimulation in a woman with residual schizophrenia. Int J Neuropsychopharmacol. 2008;11(8):1181-1183.

\section{Disclosures}

The authors report no conflict of interest concerning the materials or methods used in this study or the findings specified in this paper.

\section{Author Contributions}

Conception and design: C Zhang, Wang, Y Zhang, D Li, Liu, Sun. Acquisition of data: Wang, Y Zhang, Gong, Jin, Liu, Sun. Analysis and interpretation of data: C Zhang, Wang, Y Zhang, Gong, J Li, Jin. Drafting the article: C Zhang, Wang. Critically revising the article: C Zhang, D Li, Sun. Reviewed submitted version of manuscript: C Zhang, Wang, D Li, Sun. Approved the final version of the manuscript on behalf of all authors: C Zhang. Statistical analysis: Wang. Administrative/technical/material support: C Zhang, J Li, D Li, Liu, Sun. Study supervision: C Zhang, D Li, Liu, Sun.

\section{Supplemental Information \\ Videos}

Video 1. https://vimeo.com/418903496.

\section{Correspondence}

Chencheng Zhang: Ruijin Hospital, Shanghai Jiao Tong University School of Medicine, Shanghai, China. i@cczhang.org. 